\title{
Pathology of Resin-Induced Gastrointestinal Damage: Report of 15 Cases and Review of Literature
}

\author{
Smiley Annie GEORGE (D), Issam FRANCIS \\ Department of Histopathology, Mubarak Al Kabir Hospital, JABRIYA, KUWAIT
}

\begin{abstract}
Objective: Medication resins such as Kayexalate and Sevelamer used in the setting of chronic kidney disease for the correction of hyperkalemia and hyperphosphatemia are associated with gastrointestinal mucosal injury. In this study we describe the clinico-pathological features of Resin-induced gastrointestinal mucosal injury highlighting the histo-morphological appearances and differential diagnoses. The aim of this study is to increase the awareness of pathologists and clinicians alike to an under-reported etiology and pattern of intestinal mucosal injury related to medical resin therapy which may at times pose a clinical emergency.
\end{abstract}

Material and Method: The archives of the Department of Histopathology, Mubarak Al Kabir hospital were analyzed for cases of resin-induced gastrointestinal mucosal injury between 2013 and 2018.

Results: Of the 15 cases, Kayexalate crystals were identified in 7 cases, Sevelamer in 5 cases and both together were seen in 3 cases. Resin crystals were identified in the gastric antrum\&duodenum ( 3 cases), colon ( 9 cases in the left colon, 2 cases in the right colon) and anal canal ( 1 case). The histological tissue reactions included mucosal necrosis (1 case), inflammatory polyps (2 cases), mucosal ulcerations with granulation tissue formation (10 cases), perforation (1 case), and luminal crystals (1 case).

Conclusion: Accurate and timely recognition of the resin crystals in biopsy samples with clinical correlation is mandatory to avoid serious complications.

Key Words: Resins, Kayexalate, Sevelamer, Gastrointestinal tract injury

\section{INTRODUCTION}

The gastrointestinal tract (GIT) is a target of several types of medical drugs including nonsteroidal anti-inflammatory drugs, iron pills, Taxol, Cellcept, bisphosphonates, colchicines and oral resins used in renal failure patients (1).

Resins which include Kayexalate, Sevelamer and bile acid sequestrant agents are non-absorbable medications that facilitate ion exchange. Their main therapeutic effect is exerted within the lumen of the GIT. Reported clinical adverse effects related to the GIT include constipation, nausea, diarrhea and vomiting. In addition, mucosal ulceration including wall perforation and post-inflammatory stricture formation are among the serious complications which may present as a clinical emergency (2-12).

Pathologists are increasingly required to identify such resin-induced damage in small biopsies. This however, can be very challenging since changes reported overlap with those seen in inflammatory bowel disease, ischemic colitis, infectious colitis as well as microscopic colitis. One of the

(Turk Patoloji Derg 2019, 35:221-227)

Received : 17.02.2019 Accepted : 27.03.2019 main distinctive features of resin mucosal damage is the identification of resin crystals. This is reported in roughly in $75 \%$ of cases according to a study by Gonzalez et al. (13). It is logical to think that familiarity of the reporting pathologist with the different morphological appearances of such resins coupled with a high index of suspicion with clinical correlation is crucial.

In the current study, we present a series of 15 cases encountered in our teaching hospital in which resins were identified in various types of surgical and biopsy material of the GIT with discussion of their pathological features and correlation with other inflammatory disorders of the GIT.

\section{MATERIALS and METHODS}

A retrospective review of our archives between 2013 and 2018 uncovered 15 cases where resins were identified in biopsy materials. The Hematoxylin and Eosin (H\&E) stained sections were evaluated for general morphology. This was complemented by examination of two additional ancillary stains: Periodic Acid-Schiff with diastase (PAS/D)

Correspondence: Smiley Annie GEORGE

Department of Histopathology, Mubarak Al Kabir Hospital,

JABRIYA, KUWAIT

E-mail: annsmiley78@gmail.com Phone: +00 96598821275 
and Zeihl-Neelsen Acid-Fast Bacillus (AFB) stain. The following patient details were extracted from the electronic file: age, sex, type of specimen, clinical history provided on the request forms; patient clinical data from the case files were retrieved in addition to other laboratory results at the time of admission. These were correlated with the type of crystal seen and the associated tissue reactions in the biopsy.

Kayexalate crystals were identified by their mosaic pattern of rectangular shaped "fish scales" showing perpendicular points of intersection; they appeared purple on H\&E (Figure 1A, B), turned magenta on PAS/D (Figure 1C) and stained black with acid-fast stains (Figure 1D) $(8,13,14)$. These crystals were refractile but did not polarize.

Sevelamer crystals were non-polarizable with broad, curved, and irregularly spaced "fish scales" and displayed a 2-toned color imparted by bright pink linear accentuations and a rusty yellow background (Figure 2A,B). They acquired a violet color on PAS/D and maintained their internal structure (Figure 2C) and magenta color on AFB stain (Figure 2D) (8).

\section{RESULTS}

A total of 15 cases were analyzed for this study. The clinicopathological features are summarized in Table I. Of the 15 cases, 3 were surgical resections and 12 were biopsy samples. The patients were 10 males and 5 females and the age range was 41- 82 years. Kayexalate crystals were identified in 7 cases, Sevelamer in 5 cases and both together were seen in 3 cases. Resin crystals were identified in the upper GIT (duodenum \& gastric antrum, 3 cases) (Figure 1A) and colon (11 cases of which 9 were in the left side of the colon and 2 cases in the right side) (Figure 2A; $3 \mathrm{~A}$ ).
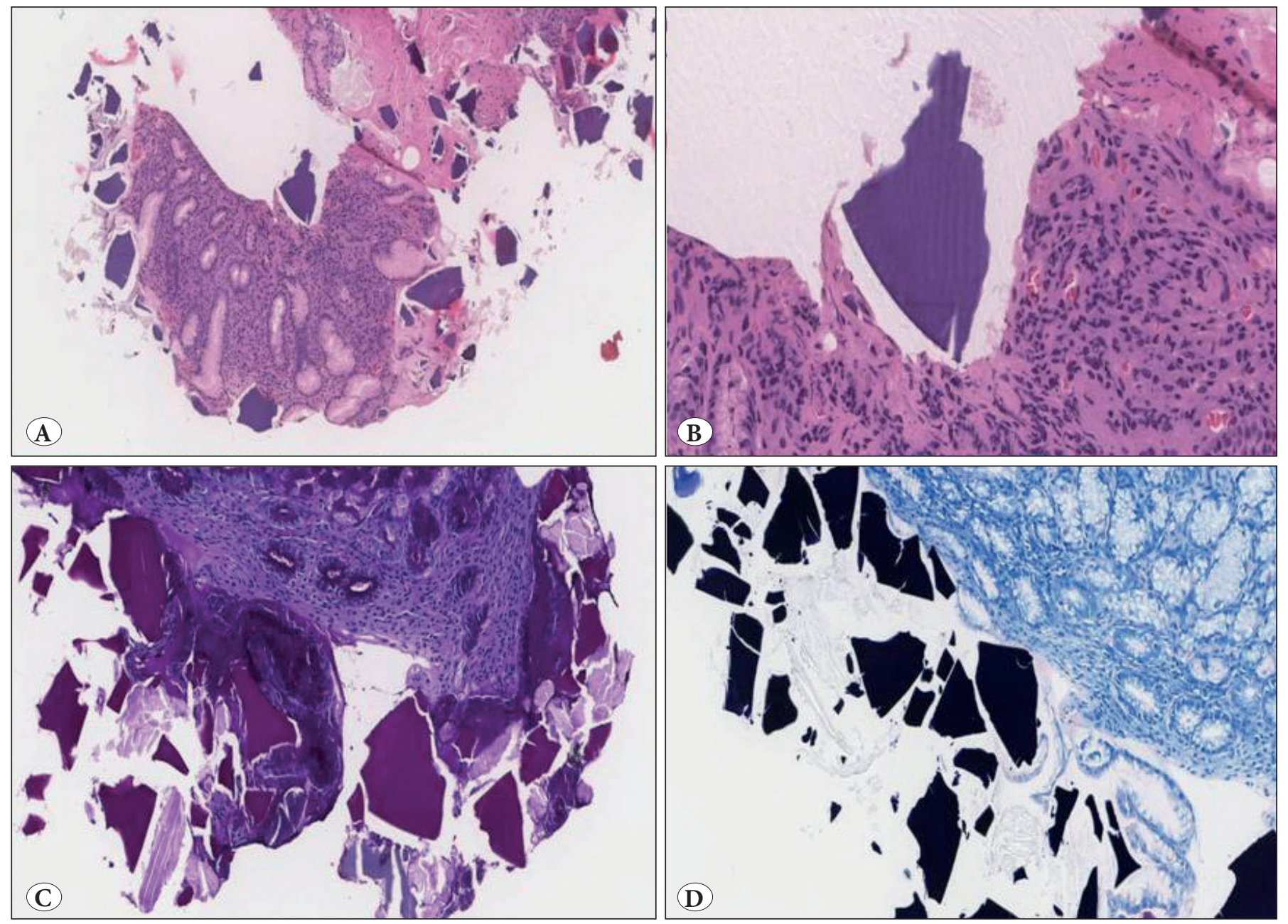

Figure 1: A) Gastric antral biopsy with ulceration and purplish Kayexalate crystals (H\&E; x50). B) Purplish Kayexalate crystals with rectangular shaped fish-scales (H\&E; x400). C) Magenta colored Kayexalate crystals (PAS/D; x200). D) Black colored Kayexalate crystals (Acid Fast stain; x200). 

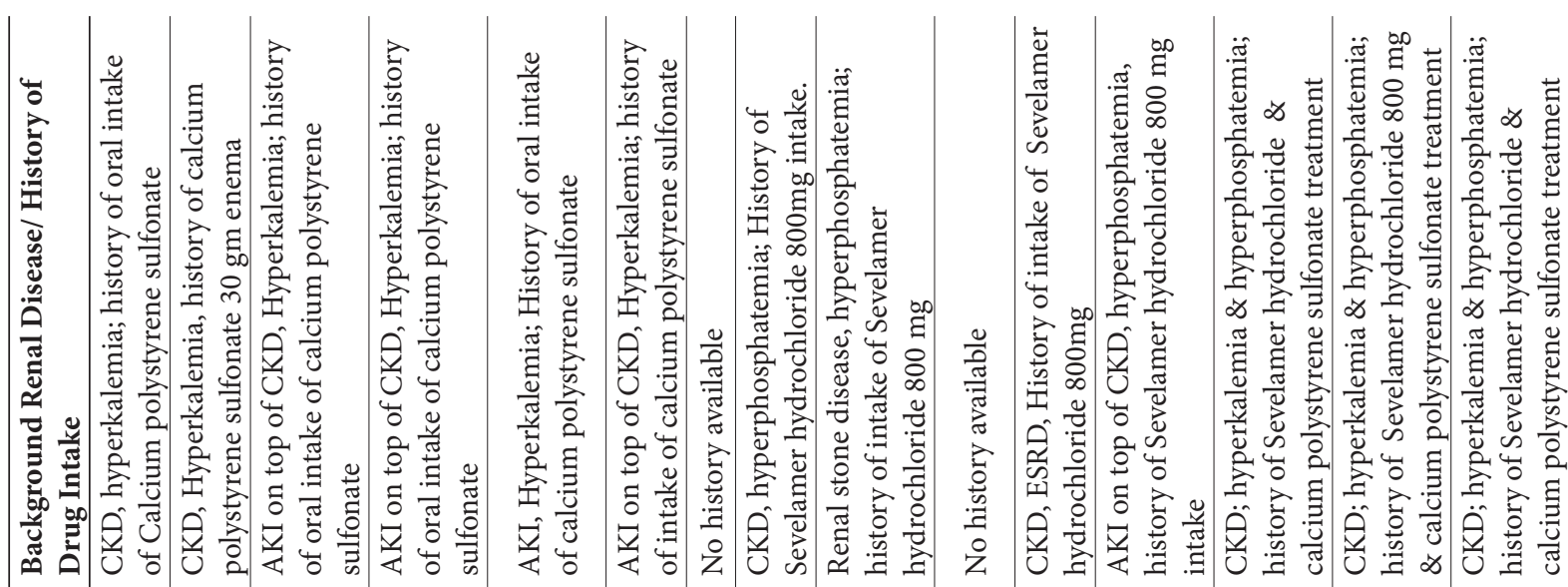

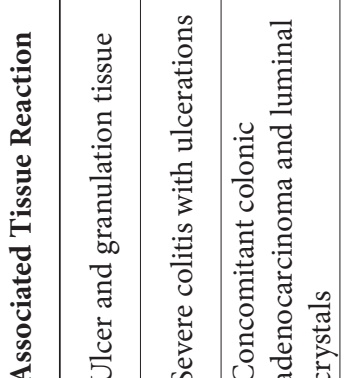

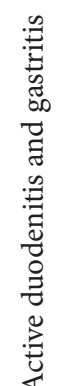
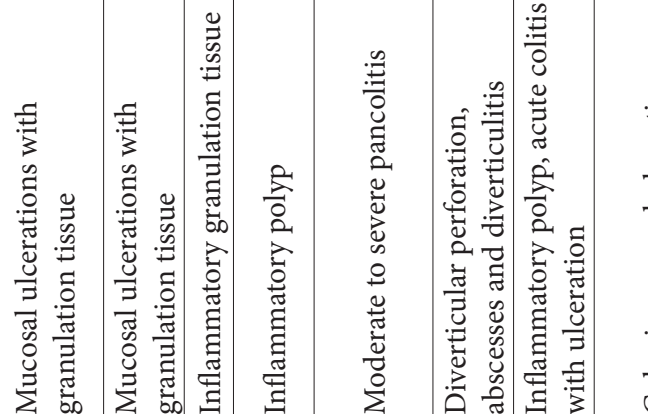

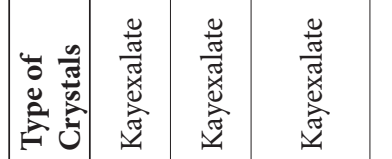

莺

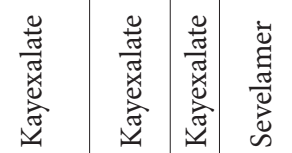

节

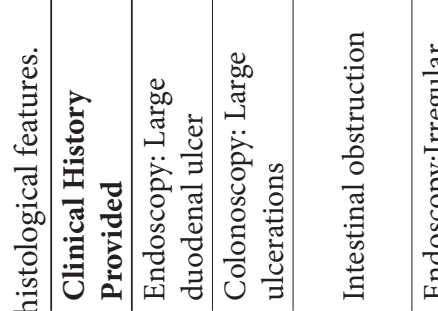

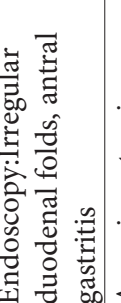

\section{范}

\section{0}

ت্ّ

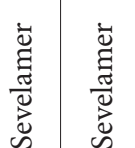

岕

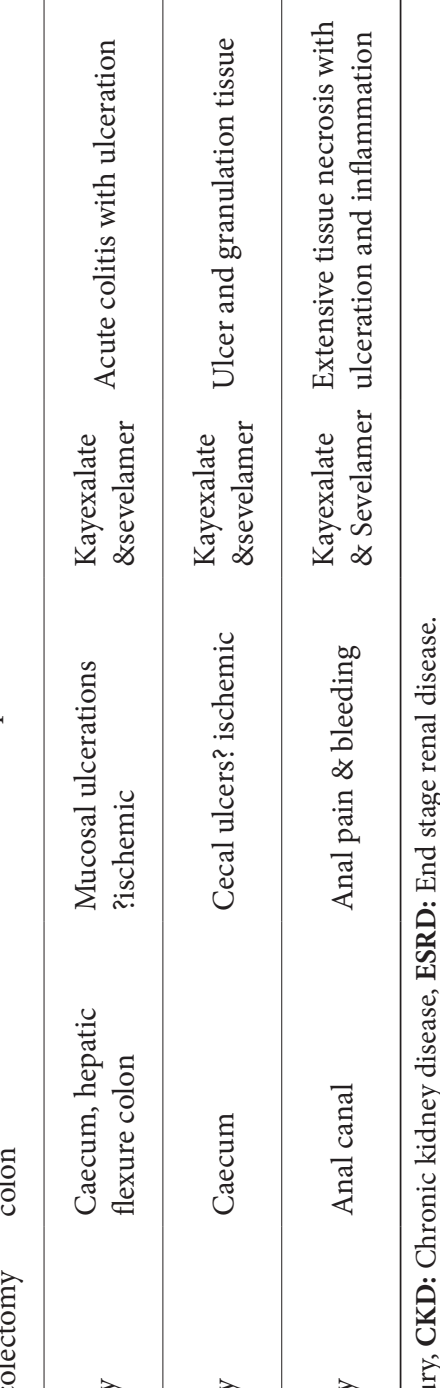

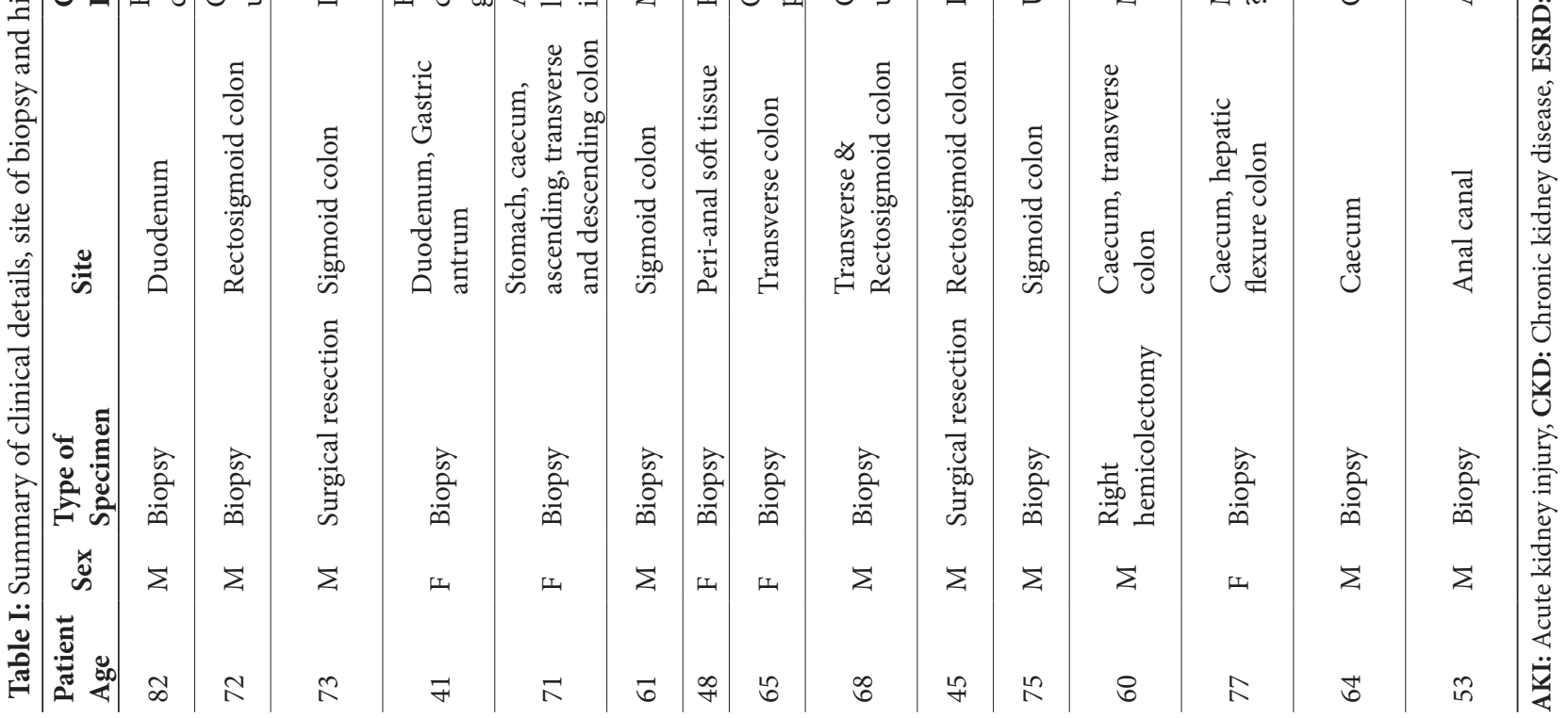


Two cases showed crystals in the perianal soft tissue associated with fistula and the other in the anal canal associated with extensive tissue necrosis (Figure 3B). Among the gastrointestinal cases, three cases had concomitant pathology such as ulcerative colitis, diverticular disease and colonic adenocarcinoma. The remainder of the cases presented as ulcerations endoscopically.

None of the pathology request forms had any relevant clinical history such as background of chronic kidney disease (CKD) or any history of intake of these resins. Retrieval of data from case files showed up background of $\mathrm{CKD}$ and relevant drug history in 13 cases. No supporting clinical histories were obtained in two cases.

\section{DISCUSSION}

Kayexalate is a non-absorbable ion-exchange resin consisting of sodium polystyrene sulfonate (SPS). It is mainly used for treating hyperkalemia in chronic renal failure and can be administered orally or locally as an enema. An osmotic laxative (Sorbitol) is usually added to reduce the risk of constipation and impaction. The gastrointestinal complications are seen more frequently in uremic patients and in post-transplant patients $(2,4,5)$. Both Kayexalate crystals and the hyperosmotic sorbitol diluent used are considered to cause marked mucosal injury (3).

Overall, the clinicopathological features associated with Kayexalate crystals in the current report are similar to
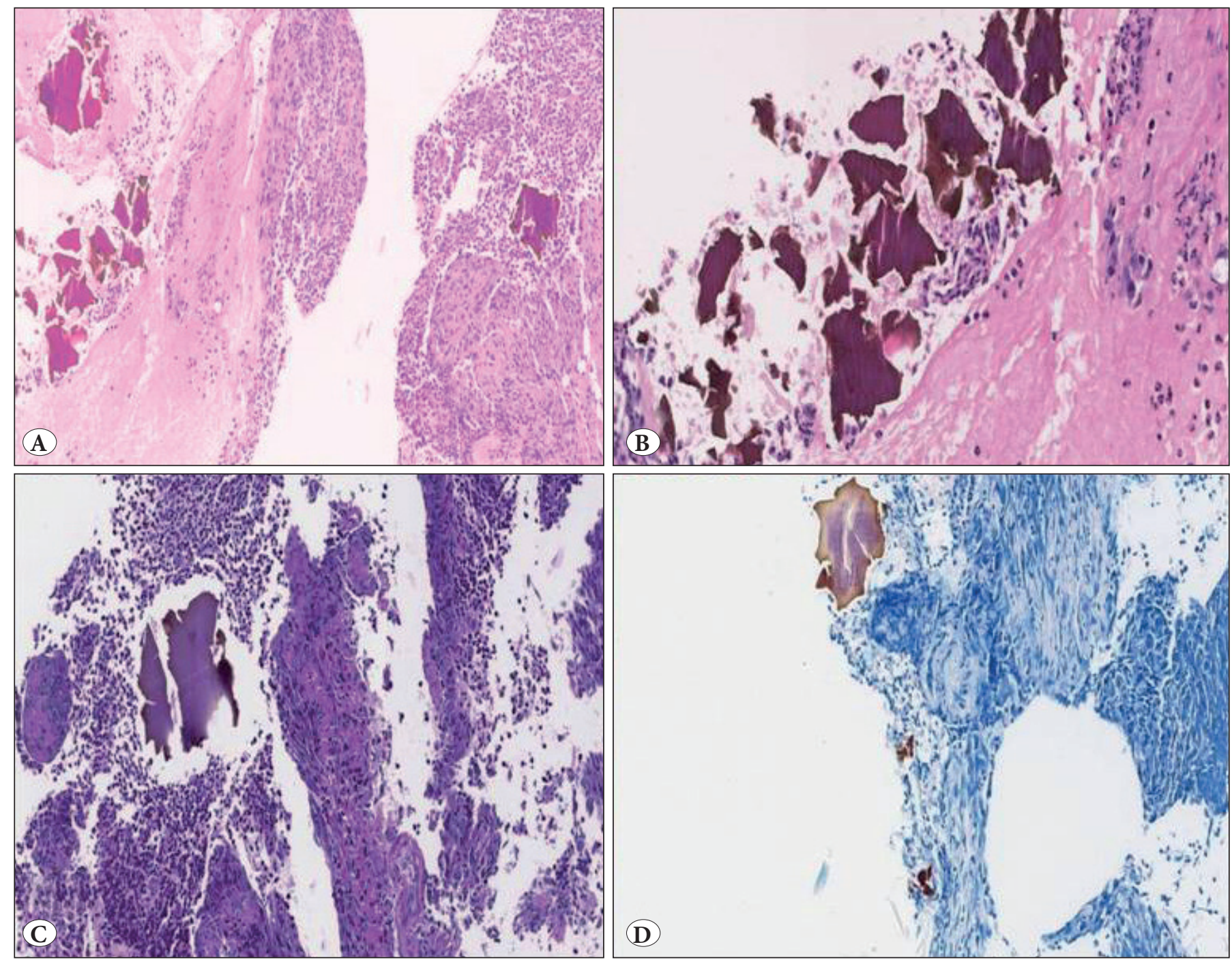

Figure 2: A) Colonic ulceration with acute inflammatory exudate and Sevelamer crystals (H\&E; x50). B) Sevelamer crystals with a 2-toned color imparted by bright pink linear accentuations and a rusty yellow background and irregularly spaced fish-scales (H\&E; x400). C) Sevelamer crystals with violet hue (PAS/D; x200). D) Magenta colored Sevelamer crystals (AFB; x200). 
those in the literature. Ulcerations were identified in 7 of the gastrointestinal biopsy cases and were associated with inflamed granulation tissue (Figure 1A, 3A). In one of the cases with concomitant adenocarcinoma, Kayexalate crystals were noted in the lumen but without any inflammatory tissue reaction.

Sevelamer, the newest in the family of resin medications, is a phosphate binding anion-exchange resin which is used to treat and prevent hyperphosphatemia caused by chronic renal failure. It is a cross-linked polymeric amine that also binds bile acids and is not systemically absorbed.

The histopathology of Sevelamer crystals in the GIT was described by Swanson et al. They noted that their presence was associated with mucosal abnormalities such as inflammation, ulceration, ischemia and necrosis
(8). Though limited, a literature search has revealed 16 case reports of Sevelamer-induced gastrointestinal complications such as recto-sigmoid ulcerations, mass forming lesions, and diverticular rupture (9-12). Our cases also showed mucosal necrosis (Figure 3B), mucosal ulcerations with granulation tissue formation (Figures 2A; 3C,D) and perforation. The mode of injury is theorized to be linked to CKD associated gastrointestinal dysmotility, abnormal secretion and absorption which potentially increase the risk of Sevelamer crystallization and deposition in tissue $(8,12)$. However larger studies are warranted for elucidating the exact mechanism of injury.

Of note, the histological tissue reactions seen in resininduced gastrointestinal injury significantly overlap with other gastrointestinal pathologies such as acute infective colitis, ischemic colitis, inflammatory bowel disease,
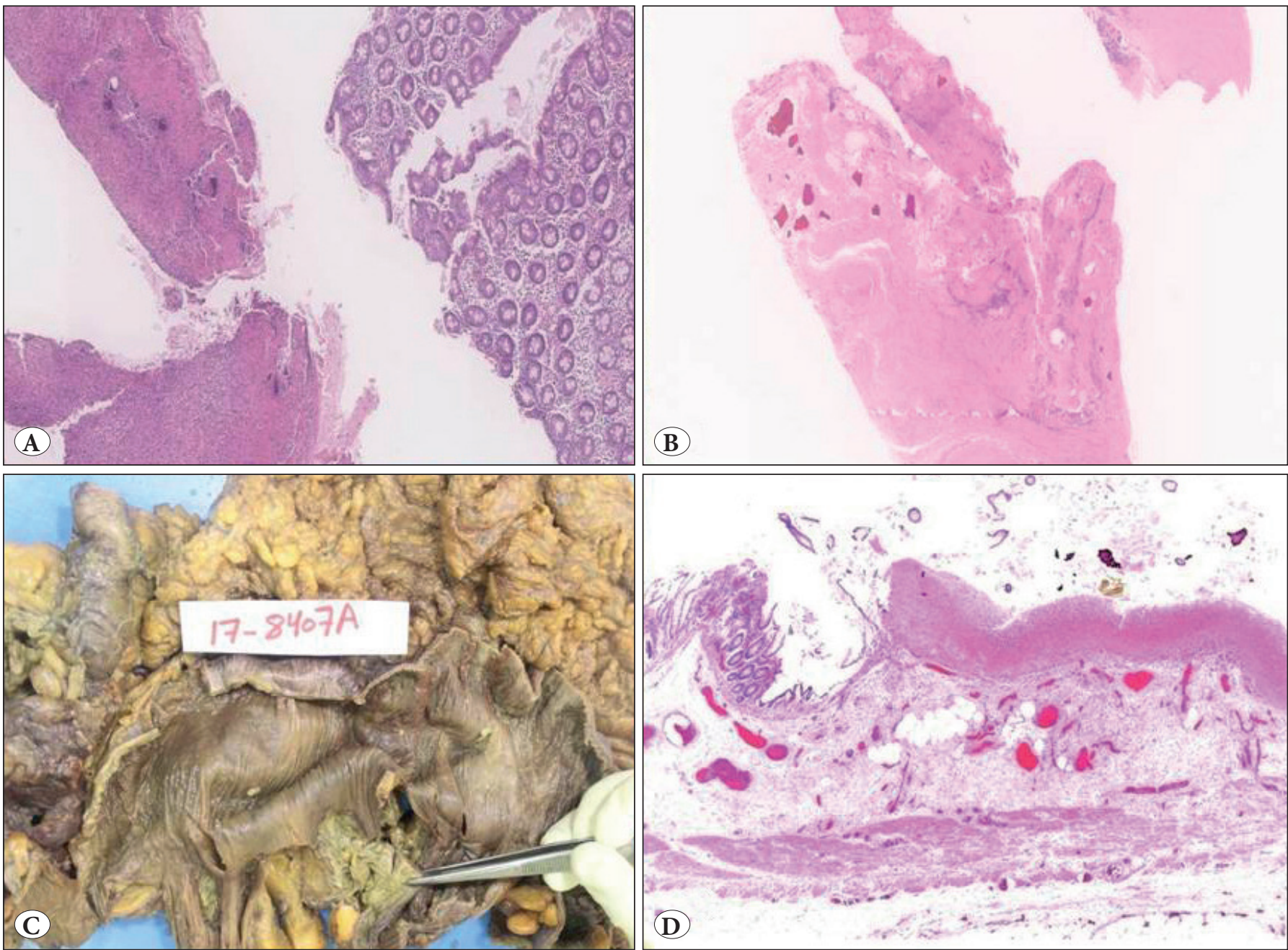

Figure 3: A) Colonic biopsy with Kayexalate induced ulceration and granulation tissue formation (H\&E; x50). B) Anal canal mucosal necrosis with Sevelamer crystals (H\&E; x200). C) Gross pathology of colonic resection with mucosal ulcerations smeared with exudates. D) Ulcerated colonic mucosa with Sevelamer crystals and acute inflammatory exudate (H\&E; x50). 
etc. Most of our cases had a clinical and/or endoscopic diagnosis of either ischemic colitis or inflammatory bowel disease. In this scenario, morphological recognition of the crystals supported by the clinical history helps in clinching the diagnosis.

Resins have been described in unusual locations and we also found them in the peri-renal soft tissue of a graft nephrectomy case and the peri-anal soft tissue in a case of peri-anal fistula. Morphological features compatible with Kayexalate crystals were identified in the peri-anal soft tissue which clinically presented as a fistula but no supporting clinical data was available in this case. The graft nephrectomy showed both Kayexalate and Sevelamer crystals admixed with suppurative inflammation in the peri-renal soft tissue. The presence of vegetable matter signaled an underlying intestinal perforation which was masked in this case (14).

Non-classical resin morphology poses problems in the accurate recognition of these crystals on H\&E. These crystals can show overlapping tinctorial qualities which are affected by the background $\mathrm{pH}$ (13). In one of our cases, Sevelamer crystals were purplish on routine stain and overlapping in appearance with Kayexalate but were magenta colored on AFB stain.
It should be noted that fish scale patterns can also be produced by dystrophic calcifications, gall bladder calculi, some food particles, large bile acid sequestrant fragments and even specimen ink used in grossing. Examining the entire histologic section as well as requesting multiple levels and special stains such as PAS/D and AFB stain can help in the accurate recognition of these crystals. Among the special stains, PAS/D stain is reported to provide inconsistent results (13). From a practical stand point, though non-classical in location and morphology, it is prudent to document the presence of the resins in biopsy reports. Histological mimics of these crystals include Cholestyramine, vegetable matter and Crospovidone. Cholestyramine is a bile acid sequestrant that is used to treat hyperlipidemia. Its crystals are rhomboid in shape and are smooth and glassy without a mosaic pattern. They stain bright orange-red on $\mathrm{H} \& \mathrm{E}$, variably gray or hot pink on PAS/D, lack internal "fish scales," and are unassociated with mucosal injury (8).

Lanthanum carbonate, a noncalcemic phosphate binder also causes gastrointestinal mucosal injury and is characterized histologically by prominent lamina propria histiocytes containing calcium like eosinophilic mineral deposition (15).
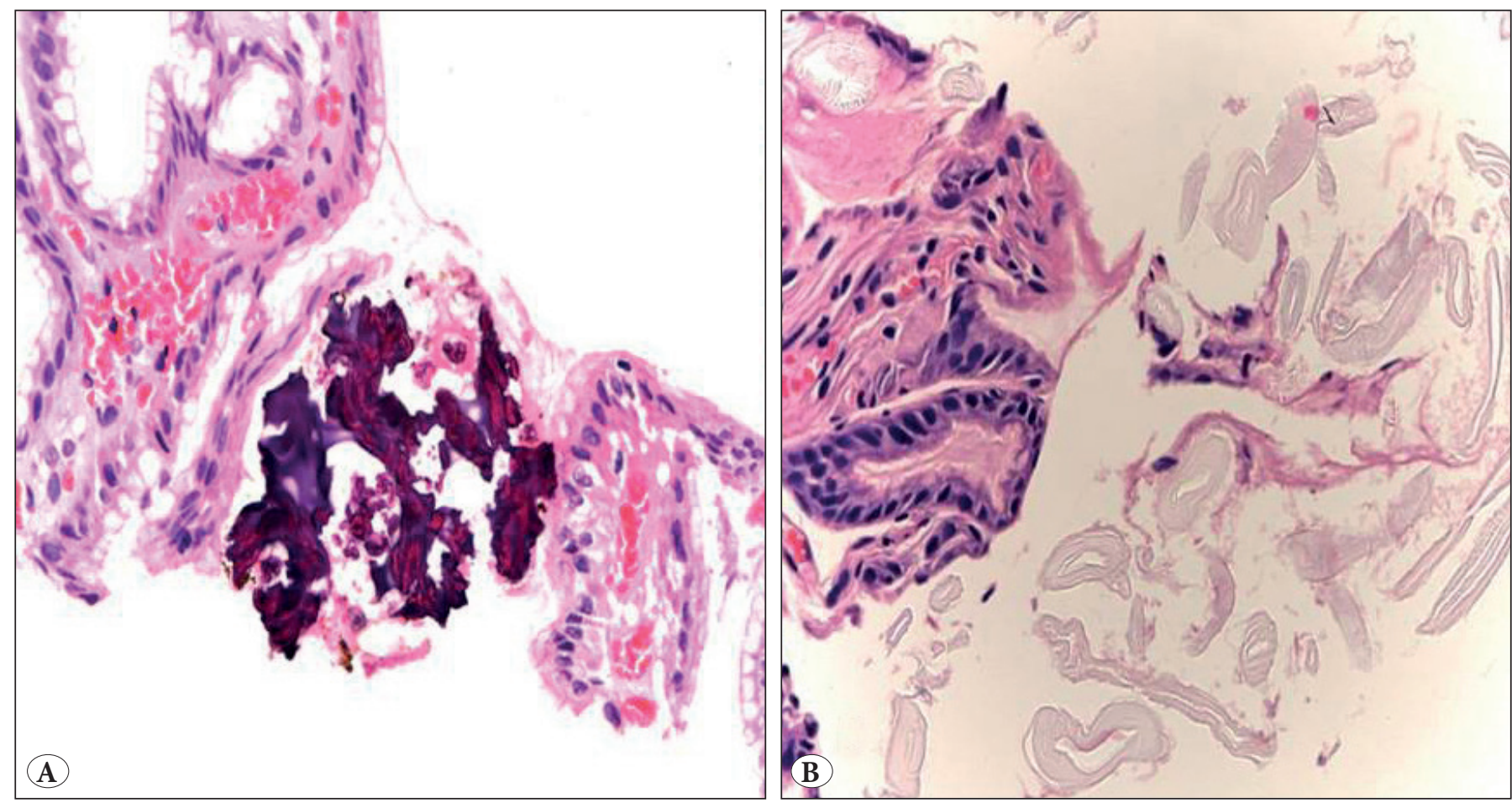

Figure 4: A) Coral shaped purplish Crospovidone crystals (H\&E; x 400). B) Matchstick shaped clear colored microcrystalline cellulose (H\&E; x 400). 
Plant or vegetable matter, which is commonly encountered in gastrointestinal biopsies, can also mimic fish-scales. However, they are usually more rectangular and often resemble "window panes". In addition, vegetable material typically will not show the characteristic colors of the aforementioned crystals.

Crospovidone and Microcrystalline cellulose can also show up in gastrointestinal biopsies but are innocent bystanders with no specific clinic-pathologic associations. They are water-insoluble, nonabsorbable pharmaceutical fillers or binders incorporated into medications (i.e. tablets) to facilitate drug delivery. Crospovidone is non-birefringent and has coral/sponge shape segments composed of a pink core and purple coat (Figure 4A). Microcrystalline cellulose is brightly birefringent, resembling cotton fibers, but with a more prominent matchstick shape and clear color (Figure 4B) (16).

Careful review of the medication history is mandatory in the definite diagnosis of these crystals. Unfortunately, usually no clinical data will be provided in the request forms. None of our cases had any clinical data and particularly drug history mentioned in the requests. Retrieval of the patient case file is helpful in these instances.

In conclusion, the aim of this study is to increase the awareness of pathologists and clinicians alike to an under-reported etiology and pattern of intestinal mucosal injury related to medical resin therapy. Resin-induced intestinal injury may at times pose a clinical emergency. Their presence in biopsy material should therefore be reported soon to the treating clinician to watch for any serious untoward consequences. The clinicians should be increasingly aware of this pattern of intestinal damage and add it to their differential diagnosis in the proper clinical setting. Pathologist must be aware of this pattern of intestinal injury and report it promptly. Availability of the complete clinical history and other laboratory tests at the time of pathological evaluation will aid in establishing the correct diagnosis.

\section{CONFLICT of INTEREST}

The authors declare no conflict of interest.

\section{REFERENCES}

1. Panarelli NC. Drug-induced injury in the gastrointestinal tract. Semin Diagn Pathol. 2014; 31:165-75.

2. Akagun T, Yazici H, Gulluoglu MG, Yegen G, Turkmen A. Colonic necrosis and perforation due to calcium polystyrene sulfonate in a uremic patient: A case report. NDT Plus. 2011;4: 402-3.
3. Lillemoe KD, Romolo JL, Hamilton SR, Pennington LR, Burdick JF, Williams GM. Intestinal necrosis due to sodium polystyrene (Kayexalate) in sorbitol enemas: Clinical and experimental support for the hypothesis. Surgery. 1987;101:267-72.

4. Joo M, Bae WK, Kim NH, Han SR. Colonic mucosal necrosis following administration of calcium polystyrene sulfonate (Kalimate) in a uremic patient. J Korean Med Sci. 2009;24: 120711.

5. Scott TR, Graham SM, Schweitzer EJ, Barlett ST. Colonic necrosis following sodium polystyrene sulfonate(Kayexalate)-sorbitol enema in a renal transplant patient; Report of a case and review of literature. Dis Colon Rectum. 1993:36:607-9.

6. Trottier V, Drolet S, Morcos MW. Ileocolic perforation secondary to sodium polystyrene sulfonate in sorbitol use: A case report. Can J Gastroenterol. 2009; 239109:689-90.

7. Rashid A, Hamilton SR. Necrosis of the gastrointestinal tract in uremic patients as a result of sodium polystyrene sulfonate (Kayexalate) in sorbitol: An under-recognized condition. Am J Surg Pathol. 1997;219:60-9.

8. Swanson BJ, Limketkai BN, Liu TC, Montgomery E, Nazari K, Park JY, Santangelo WC, Torbenson MS, Voltaggio L, Yearsley MM, Arnold CA. Sevelamer crystals in the gastrointestinal tract (GIT): A new entity associated with mucosal injury. Am J Surg Pathol. 2013; 37:1686-93.

9. Yamaguchi T, Ohyama S,Furukawa H, Sato N, Ohnishi I, Kasashima S, Kawashima A, Kayahara M. Sigmoid colon diverticula perforation associated with sevelamer hydrochloride administration: A case report. Ann Med Surg (LOND). 2016;10:57-60

10. Okwara C, Choi C, Park JY. Sevelamer-induced colitis presenting as a pseudotumour. Clin Gastroenterol Hepatol. 2015;13:A39-A40.

11. Tieu C, Moreira RK, Song LM, Majumder S, Ppadakis KA, Hogan MC. A case report of sevelamer-associated recto-sigmoid ulcers. BMC Gastroenterol. 2016;16:20.

12. Yuste C, Merida E, Hernandez E, Garcia-Santiago A, Rodriguez Y, Munoz T, Gomez GJ, Sevillano A, Praga M. Gastrointestinal complications induced by sevelamer crystals. Clin Kidney J. 2017;10:539-44

13. Gonzalez RS, Lagana SM, Szeto O, Arnold CA. Challenges in diagnosing medication resins in surgical pathology specimens: A crystal-clear review guide. Arch Pathol Lab Med. 2017;141:127682.

14. George SA, Francis I. "Fish-scales" and graft nephrectomy: Unexpected findings at an unusual site. J Nephropathol. 2017;6:34951.

15. Khurram M, Montogomery E. Lanthanum carbonate-associated injury to the small intestine. Diagnostic Histopathology. 2015;21:452-4.

16. Shaddy SM, Arnold MA, Shilo K, Frankel WL, Harzman AE Stanich PP, Singhi AD, Yearsley MM, Arnold CA. Crospovidone and microcrystalline cellulose: A novel description of pharmaceutical fillers in the gastrointestinal tract. Am J Surg Pathol. 2017;41:564-9. 